\title{
VARIETIES OF TOPOLOGICAL GEOMETRIES
}

\author{
HANSJOACHIM GROH
}

\begin{abstract}
A variety of topological geometries is either
A. a projective variety $\mathscr{L}(F)$ over some topological field $F$, or

B. a matchstick variety $\mathscr{M}(X)$ over some topological space $X$. As a main tool for showing this, we prove a structure theorem for arbitrary topological geometries.
\end{abstract}

\section{INTRODUCTION.}

a. The Euclidean plane, the real hyperbolic plane, the real affine 3-space, and the real projective $n$-spaces are all strongly interrelated by forming subgeometries and contractions. Therefore it seems natural to consider classes of geometries. In [ $\mathrm{KK} \mathrm{82}$ ] (reprinted in [ $\mathrm{Ku} \mathrm{86]),} \mathrm{Kahn} \mathrm{and} \mathrm{Kung} \mathrm{defined} \mathrm{the}$ notion "variety of (combinatorial) geometries". They succeeded in determining all varieties of finite geometries. On p. 498 they list some difficulties associated with the determination of all varieties of arbitrary geometries. Therefore it seems reasonable to add an additional structure: In this paper we determine all varieties of topological geometries:

5.4. Main Theorem II. Let $\mathscr{T}$ be a variety of topological geometries containing a nondiscrete one. Then $\mathscr{T}$ is either

A. a projective variety $\mathscr{L}(F)$ over some topological field $F$, or

B. a matchstick variety $\mathscr{M}(X)$ over some Hausdorff topological space $X$.

Comparing this with the finite case we observe that none of the three types: free variety, origami varieties, voltage-graphic varieties, has a topological analogue. If we view matchstick geometries as trivial, then 5.4 even says that only "classical" geometries (i.e. geometries coordinatizable over some topological field) can be members of a variety. Furthermore, of the multitude of nondesarguesian topological projective planes, none belongs to a variety.

There exist projective as well as matchstick varieties admitting more than one sequence of universal models:

A. Let $\mathbb{R}(x)$ be the field of rational functions over $\mathbb{R}$ (reals). There is a linear ordering on it with $r<x$ for all $r \in \mathbb{R}$, which makes it an ordered field. Endowed with the open interval topology induced by this ordering, $\mathbb{R}(x)$ becomes a topological field $F . \mathbb{R}\left(x^{2}\right)$ is a proper subfield isomorphic to $F$. Therefore (see [KK 82, p. 498]), defining $\mathbf{T}_{n}$ to be the affine $n$-space over

Received by the editors December 20, 1989 and, in revised form, February 18, 1991.

1980 Mathematics Subject Classification (1985 Revision). Primary 05B35; Secondary 51H10. 
$F$, we obtain another sequence of universal models for the projective variety $\mathscr{L}(F)$.

B. Let $X=\mathbb{R}$ and $Y:=[0,1]$, the closed unit interval. Since $X$ is homeomorphic to the subspace ]0, 1 [ of $Y$, by 5.3 we have $\mathscr{M}(X)=\mathscr{M}(Y)$. Therefore the matchstick variety $\mathscr{M}(X)$ has at least two sequences of universal models: The powers of the line over $\mathbb{R}$, and the powers of the line over $[0,1]$.

Our proof for the Main Theorem II is not analogous to the corresponding one in [KK 82]. We derive it from a structure theorem on arbitrary topological geometries, which is of interest in its own right:

4.6. Main Theorem I. Each topological geometry $\mathbf{G}$ is isomorphic to the direct product of finitely many

(1) open subgeometries of projective spaces over topological fields,

(2) topological planes (not necessarily desarguesian or projective),

(3) lines over Hausdorff topological spaces, or

(4) discrete geometries.

\section{b. Notions. Notation. Facts.}

b1. Let $\mathbf{G}$ be a geometric lattice (also called geometry, or matroid; see e.g. [Bi 67, CR70, Ku 86, We 76, Wh 86, Wh 87]). For $x \in \mathbf{G}$, we define the dimension of $x$-denoted by $\operatorname{dim} x$-to be the cardinality of a maximal chain from the smallest element 0 to $x$, minus 2 . The dimension of $\mathbf{G}$ is defined by $\operatorname{dim} \mathbf{G}:=\operatorname{dim} 1=: n$, where 1 is the largest element. Elements of dimension $0,1,2, k, n-2, n-1$ are called points, lines, planes, $k$-flats, colines, hyperplanes (or copoints). Furthermore, if $n=0,1,2$, resp. $k$, then $\mathbf{G}$ itself is called a point, line, plane, resp. $k$-space.

For $x \in \mathbf{G}$, the intervals $\mathbf{G}_{x}:=[0, x]$ and $\mathbf{G}^{x}:=[x, 1]$ (contraction) are again geometric lattices, as is the following: For a set $M$ of points, the subgeometry $\mathbf{G}(M)$ induced on $M$ is the set of flats of $\mathbf{G}$ for which $M$ contains a spanning set, with the ordering inherited from $\mathbf{G}$. Furthermore we use the following symbols:

$$
\begin{array}{ll}
x \lessdot y & \text { for: } x \text { is a lower neighbor of } y, \\
& \text { set of upper neighbors of } x, \\
x & \text { set of points below } x, \\
{ }_{0} & \text { set of } k \text {-flats of } \mathbf{G}, \\
{ }_{k} \mathbf{G} & \text { restriction of } \bigvee \text { to }\left\{(x, y) \in{ }_{i} \mathbf{G} \times{ }_{j} \mathbf{G} \mid x \vee y \in{ }_{k} \mathbf{G}\right\}, \\
{ }_{i}^{j} \bigvee_{k} & \text { is defined dually. } \\
{ }^{i} \bigwedge^{k} &
\end{array}
$$

b2. A topological geometry (or topological $n$-space) is a geometric lattice $\mathbf{G}$ where each "layer" ${ }_{j} \mathbf{G}$ of $j$-flats carries a topology such that

(CV) Joining a point with a $j$-flat is continuous.

$(\mathrm{C} \wedge)$ Meeting a hyperplane with a $j$-flat resulting in a $(j-1)$-flat, is continuous.

(S) Each hyperplane $h$ and $j$-flat $x$ with $j \geq 1$ meeting in a $(j-1)$-flat, have neighborhoods $V$, resp. $W$, with the same properties, i.e. each $h^{\prime} \in V$ meets each $x^{\prime} \in W$ in a $(j-1)$-flat. 
This notion has been justified in [ $\mathrm{Gr}$ 86a]. Furthermore, in [CG 89] it is shown that it would be too strong to postulate-as the analogy to topological groups etc. might suggest it-a topology on $\mathbf{G}$ such that the "full" functions of $\vee$ and $\wedge$ are continuous. Instead of postulating this, our three axioms only require all "slices" $0 \bigvee_{j}^{j+1}$ and ${ }^{n-1} \bigwedge_{j-1}^{j}$ to be continuous, and the domain of ${ }^{n-1} \bigwedge_{j-1}^{j}$ to be open.

We define the topology $\tau$ of $\mathbf{G}$ to be that of the free union of the ${ }_{j} \mathbf{G}$ (cf. [Gr 86a, p. 115]). Thus, a topological geometry could equivalently be defined as a pair $(\mathbf{G}, \tau)$ of a geometric lattice and a topology, satisfying $(\mathrm{C} \vee),(\mathrm{C} \wedge)$ and (S), where each $j \mathbf{G}$ is open.

For a topological field $F$, the topological projective $n$-space over $F$, denoted by $\mathbf{P}_{n}(F)=: \mathbf{P}$, is the lattice of subspaces of the $F$-vector space $F^{n+1}$, given the following topology $\tau$ : For $M \subseteq F^{n+1}$, denote by $M_{\mathbf{P}}$ the set of subspaces meeting $M$. Define $\tau$ to be the topology having as a subbasis

$$
\left\{M_{\mathbf{P}} \mid M \text { open in } F^{n+1}\right\} \cup\left\{{ }_{i} \mathbf{P} \mid-1 \leq i \leq n\right\} .
$$

From [Sz 86, Proposition 3] it follows easily that $(\mathbf{P}, \tau)$ is a topological projective space in the sense of [Mi 68, p. 246], which by [Gr 86a, Theorem 3.3] is a topological geometry.

b3. The (direct) product $\mathbf{G}_{1} \times \mathbf{G}_{2}$ of two topological geometries $\mathbf{G}_{i}$ is defined as the (direct) product (see e.g. [Bi 67, p. 8]) of the corresponding lattices, endowed with the product topology. Since a variety is closed under the formation of products, it is important to observe the following fact:

1.1. The product of two topological geometries is again a topological geometry.

The proof is a verification using generalized sequences (or nets, see e.g. [Du 66, p. 2.10]).

b4. We write $\cong$ for "isomorphic" (see [Gr 86a, p. 115]), and $\approx$ for "homeomorphic".

\section{Planes}

The following proposition is a generalization of [ $\mathrm{Gr} 86 \mathrm{a}$, Corollary 5.3]. It is needed here for planes only. However, the proof for this special case would not be simpler.

2.1. Let $\mathbf{G}$ be an arbitrary topological geometry. Let $h_{i}$ be two hyperplanes, I a line such that $h_{i} \wedge l=: p_{i}$ are points. If either

a. $l$ contains at least three points, or

b. $p_{1}=p_{2}$,

then there exist neighborhoods $U_{i}$ of $p_{i}$ in $\stackrel{\circ}{h}_{i}$ with $U_{1} \approx U_{2}$.

Proof. By (S) there exists a neighborhood $V$ of $l$ all of whose lines meet each $h_{i}$ in a point. In both cases $\mathbf{a}$ and $\mathbf{b}, l$ contains a point $q$ different from both $p_{i}$. Since $V \cap q$ is open in the set $q$ of all lines through $q$, by [Gr 86a, Lemma 4.3] the set

$$
U:=\overbrace{V \cap q}^{\circ} \backslash\{q\}
$$


of all points $\neq q$ on these lines is an open point set. Now the sets $U_{i}:=U \cap \stackrel{\circ}{h}_{i}$ are the required neighborhoods, and $p_{1}^{\prime} \mapsto\left(p_{1}^{\prime} \vee q\right) \wedge h_{2}$ is a homeomorphism from $U_{1}$ onto $U_{2}$.

2.2. Corollary. Let $\mathbf{G}$ be an arbitrary topological plane. Let l be a line. Let $p_{i}$ be two points on $l$ such that there exists a point $q$ outside $l$ satisfying.

(i) Each line $p_{i} \vee q$ contains at least three points.

(ii) At least three lines pass through $q$.

Then there exist neighborhoods $U_{i}$ of $p_{i}$ in $\stackrel{\circ}{l}$ with $U_{1} \approx U_{2}$.

Proof. Apply 2.1a, 2.1b, and then again 2.1a.

For the remainder of $\S 2$, let $\mathbf{G}$ be an arbitrary topological plane.

\subsection{Let $p$ be a cluster point. Then:}

a. For at least one line $l$ through $p, p$ is a cluster point in the space $\stackrel{\circ}{l}$ of all points on $l$.

b. If at least three lines pass through $p$, then $p$ is a cluster point on each line through $p$.

Proof. a. Our assumption implies the existence of a generalized sequence (or net; see e.g. [Du 66, p. 210]) $p_{\nu} \rightarrow p, p_{\nu} \neq p$. Because of $\operatorname{dim} \mathbf{G} \geq 2$ there exists a point $q$ outside $l$. Since lines are hyperplanes, by (S) $p_{\nu} \vee q=: m_{\nu}$ finally meets $l$ in a point $p_{\nu}$. Now $(\mathrm{C} \vee)$ and $(\mathrm{C} \wedge)$ imply $p_{\nu} \rightarrow p$.

Case 1. There exist arbitrarily large $\nu$ with $p_{\nu}^{\prime} \neq p$. Then $p$ is a cluster point of $\stackrel{\circ}{l}$.

Case 2. $p_{\nu}^{\prime}=p$ finally. Then $p_{\nu}^{\prime} \lessdot m:=p \vee q$ finally. Hence $p$ is a cluster point of $\stackrel{\circ}{m}$.

b. Because of a, there exists a line $l$ through $p$ containing a generalized sequence $p_{\nu} \rightarrow p$ with $p_{\nu} \neq p$. Let $l^{\prime} \neq l$ be any line through $p$. By assumption there exists a point $q$ outside $l$ and $l^{\prime}$. Analogously to the proof for a, define $p_{\nu}^{\prime}:=\left(p_{\nu} \vee q\right) \wedge l^{\prime}$. Again by $(\mathrm{S}), p_{\nu}^{\prime}$ finally is a point, and by $(\mathrm{C} \vee)$ and $(\mathrm{C} \wedge), p_{\nu}^{\prime} \rightarrow p$. By construction, $p_{\nu}^{\prime} \neq p$.

2.4. Let $l \neq m$ be two lines meeting in a point $p$ such that

(1) $p$ is a cluster point on $l$,

(2) $m$ contains at least three points.

Then each point of $l$ is a cluster point on $l$.

Proof. Let $p^{\prime}$ be a second point on $l$. Our statement will follow from Corollary 2.2, applied to $p_{1}=p, p_{2}=p^{\prime}$, and $q=$ second point on $m$. We have to show conditions (i) and (ii):

We conclude (ii) from (1) using: (a) The point space of a topological geometry is Hausdorff [ $\mathrm{Gr} 86 \mathrm{a}, 4.2]$. (b) A $T_{1}$-space having a cluster point is infinite.

To show (i) we use (2) and choose a third point $q^{\prime}$ on $m$. By (1), and (a), (b) above there exists a generalized sequence $p_{\nu} \neq p, p^{\prime}$ on $l$ with $p_{\nu} \rightarrow p$. By $(\mathrm{C} \vee)$ and $(\mathrm{S})$ the line $q^{\prime} \vee p_{\nu}$ finally meets $p^{\prime} \vee q$ in a point, which must be different from $q$ and $p^{\prime}$.

2.5. Theorem. Let $\mathbf{G}$ be a nondiscrete topological plane containing a two-point line. Then $\mathbf{G}$ contains a line $l$ and a point $q$ such that $\mathbf{G} \cong \mathbf{G}_{l} \times \mathbf{G}_{q}$. 
Proof. Since $\mathbf{G}$ is not discrete, by [Gr 86a, 4.19] the point space ${ }_{\circ} \mathbf{G}$ is not discrete, and therefore contains a cluster point $p$. By 2.3a, there exists a line $l$ through $p$ such that $p$ is a cluster point on $l$. We prove 2.5 by showing: If the plane $\mathbf{G}$ contains at least two points outside $l$ then it contains no two-point line. Actually we show that then each line has a cluster point on it, and hence (see 2.4, proof for (ii)) is infinite.

Assume $\mathbf{G}$ contains at least two points outside $l$. Either these are collinear with $p$, or there exist at least two lines $\neq l$ through $p$, and then by $2.3 \mathrm{~b} l$ is a cluster point on each line through $p$. Hence in either case, there exists a line $m \neq l$ through $p$ containing at least three points.

Therefore by 2.4 each point of $l$ is a cluster point on $l$. Let now $l^{\prime}$ be any line meeting $l$ in a point $p^{\prime} \neq p$. Joining $p^{\prime}$ with the points of $m$ we obtain at least three lines through $p^{\prime}$. Hence by $2.3 \mathrm{~b}, p^{\prime}$ is a cluster point also on $l^{\prime}$. Since $l$ contains at least three points, by 2.4 each point of $l^{\prime}$ is a cluster point on it. Finally, let $l^{\prime}$ be any line $\neq l$ either meeting $l$ in $p$, or disjoint to $l$ (i.e. $l^{\prime} \wedge l=0$ ). Choose a point $q \neq p$ on $l^{\prime}$ and two points $p_{i} \neq p$ on $l$. By the preceding paragraph, $q$ is a cluster point on $p_{1} \vee q$, and hence by $2.3 \mathrm{~b}$ on $l^{\prime}$.

\section{Regular flats. Separators}

In each following section of this paper, $\mathbf{G}$ denotes an arbitrary topological geometry, of (lattice theoretical) dimension $n$.

For 3.3 we need the following fact:

3.1. Assume that $(*)$ each line contains at least three points. If there exist a $k$-flat $z$ with $0 \leq k \leq n-2$, and an open set $W \neq \varnothing$ of upper neighbors, then $\mathbf{G}$ is discrete.

Proof. Choose $y \in W$. Because of $k \neq n-1$ there exists a hyperplane $h$ with $y \wedge h=z$. Because of $k \geq 0$ and (S) we may assume that each $y^{\prime} \in W$ meets $h$ in a $k$-flat, too. Since $z \leq y^{\prime}$, this $k$-flat must be $z$. By [Gr 86a, 4.6] the set $V$ of $k$-flats contained in some $(k+1)$-flat of $W$ is open. Since $V \cap \mathbf{G}_{h}=\{z\}$ is open in the topological geometry [Gr 86a, 4.20a] $\mathbf{G}_{h}$, by $(*)$ and $[\mathrm{Gr} 86 \mathrm{a}$, Theorem $5.4(3) \Rightarrow(6)], \mathbf{G}_{h}$ is discrete. Hence by [Gr 86 , Theorem $5.4(2) \Rightarrow(6)], \mathbf{G}$ is discrete.

Definition. A flat $x$ is regular if and only if

(*) Each line $l \leq x$ contains at least three points, and

(**) The space $\stackrel{\circ}{x}$ of points below $x$ is not discrete.

$\mathbf{G}$ is called regular if and only if 1 is a regular flat.

3.2. If $x$ is a regular flat, then:

a. $\operatorname{dim} x \geq 1$. $\stackrel{\circ}{l}$.

b. If $\operatorname{dim} x \geq 2$ then for each line $l \leq x$, each point of $l$ is a cluster point of

c. If $y \leq x$ and $\operatorname{dim} y \geq 1$ then $y$ is regular.

Proof. a: (**). b: [Gr 86a, Theorem $5.4(2) \Rightarrow(5)]$. c. Case 1: $\operatorname{dim} x=1$. Then $y=x$. Case $2: \operatorname{dim} x \geq 2:(*)$ clearly carries over to smaller flats. $(* *)$ : $y$ contains a line $l$, which by $\mathrm{b}$ has a cluster point. 
The following theorem is needed for 3.4. It generalizes [ $\mathrm{Gr} 86 \mathrm{~b}, 3.2]$. It also allows an alternative proof for [ $\mathrm{Gr} 86 \mathrm{~b}$, Theorem 3.3] not using [Ka 74, p. 176, Lemma].

3.3. Theorem. Each hyperplane $h$ and each regular j-flat $x \not \leq h$, both passing through a common point, meet in a $(j-1)$-flat.

Proof. Because of $x \not \leq h$ there exists a flat $y$ satisfying $h \wedge x=: z \lessdot y \leq x$ and hence $h \wedge y=z$. Since $\operatorname{dim} z=: k \geq 0$, by (S) there exists a neighborhood $V$ of $y$ all of whose elements meet $h$ in a $k$-flat, too. In the topological $j$ space $\mathbf{G}_{x}$, the set $W:=V \cap \mathbf{G}_{x}$ is a neighborhood of $y$ all of whose elements pass through $z$. If our statement were false, i.e. $y<x$, then because of $(*)$, 3.1 would be applicable, implying that $\stackrel{\circ}{x}$ would be discrete, a contradiction to $(* *)$.

3.4. If $\mathbf{G}$ contains a regular hyperplane $x$ and at least two points outside then it is regular.

Proof. We proceed indirectly by induction. By $3.2 \mathrm{a}$, the statement makes sense only for $\operatorname{dim} \mathbf{G} \geq 2$. Theorem 2.5 implies that it is true for $\operatorname{dim} \mathbf{G}=2$. Assume that it is true for all topological geometries of dimension $n-1$, and that there exists a $\mathbf{G}$ of dimension $n$ for which it is false. Then, since (**) carries over from $x$ to $1,(*)$ must be violated. Thus $\mathbf{G}$ must contain a two-point line $l$. Since $x$ is regular, $l \not \leq x$.

Case 1: $l \wedge x=0$. Because $n \geq 3$, there exists a hyperplane $h \geq l$ meeting $x$.

Case 2: $l \wedge x$ is a point $p$. Then $l$ contains only one further point $p^{\prime} \not \leq x$. By the assumption of 3.4, there exists a second point $q \not \leq x$. Again, because $n \geq 3$, there exists a hyperplane $h \geq l \vee q$.

In either case, Theorem 3.3 implies that $h \wedge x=: x^{\prime}$ is a regular (by 3.2c) hyperplane of the topological $(n-1)$-space $\mathbf{G}_{h}$. Since $\mathbf{G}_{h}$ contains at least two points outside $x^{\prime}$, it is regular by our induction hypothesis. In particular, $l \leq h$ contains at least three points, a contradiction.

3.5. Corollary. If $x$ and $y$ are regular flats with $x \wedge y \neq 0$, then $x \vee y$ is regular.

Proof. We may assume $y \leq x$. Then because of $x \wedge y \neq 0$, there exists a line $l \leq y$ such that $x \wedge l$ is a point. Define $x_{1}:=x \vee l$. In the topological geometry $\mathbf{G}_{x_{1}}, x$ is a regular hyperplane. Since $y$ is regular, $l$ contains at least two points outside $x$. Hence by $3.4, x_{1}$ is regular. This proves our statement in case $x_{1}=x \vee y$.

In case $x_{1}<x \vee y$, we repeat the above procedure: $x_{2}:=x_{1} \vee l_{1}, \ldots, x_{k}:=$ $x_{k-1} \vee l_{k-1}=x \vee y$.

3.6. Assume $\mathbf{G}$ contains a maximal regular flat $x<1$. Let $h$ be a hyperplane satisfying $0<x \wedge h=: y<x$. In case $x$ is a line, assume further: (c) $y$ is a cluster point on $\stackrel{\circ}{x}$. Then $\mathbf{G}$ contains no point outside $x$ and $h$.

Proof (indirect; see Figure 1). Assume there exists a point $q \notin \stackrel{\circ}{x} \cup \stackrel{\circ}{h}$. By assumption, there exists a point $p \leq y$. Because of $y<x$ there exists a line $l \leq x$ with $l \wedge y=p$. Since $x$ is regular, by $3.2 \mathrm{~b}-$ in case $x$ is a line by 


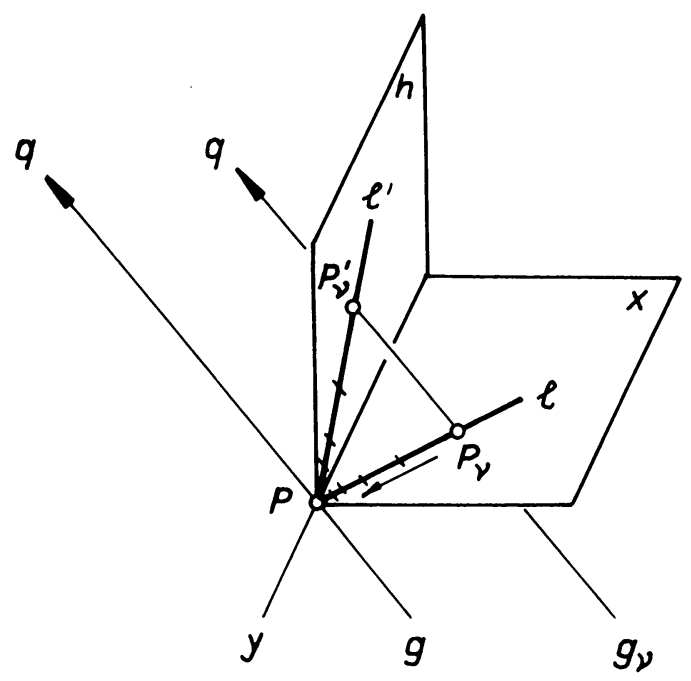

FIGURE 1

(c) 一there exists a generalized sequence $p \neq p_{\nu} \lessdot l$ converging to $p$. Because of $q \not \leq h, q \vee p=: g$ is a line meeting $h$ in a point (namely $p$ ). By $(\mathrm{CV})$ and (S), $q \vee p_{\nu}=: g_{\nu}$ is a line finally meeting $h$ in a point $p_{\nu}^{\prime}$. Because of $q \not \leq x$ we have $p_{\nu}^{\prime} \neq p$. Hence $(q \vee l) \wedge h=: l^{\prime}$ is a line containing the generalized sequence $p_{\nu}^{\prime}$, which by $(\mathrm{C} \wedge)$ has $p$ as a cluster point. Now $l^{\prime} \leq x$ implies $x^{\prime}:=x \vee l^{\prime}>x$. By Corollary 3.5, $x^{\prime}$ is regular, too, a contradiction to the maximality of $x$.

3.7. Assume $\mathbf{G}$ contains a maximal regular flat $l<1$ which is a line. Then there exists a coline $y$ satisfying

(1) $l \wedge y=0$,

(2) G contains no point outside $l$ and $y$.

Proof. By $(* *), \stackrel{\circ}{l}$ contains a cluster point $p$. Because of $l<1$ there exists a hyperplane $h$ with $l \wedge h=p$. Choose $y \lessdot h$ with $p \not \leq y$. Clearly, $y$ satisfies (1). (2) (Indirect; see Figure 2): Assume there exists a point $q$ neither on $l$ nor on $y$. By 3.6 we have ${ }_{\circ} \mathbf{G}=\stackrel{\circ}{l} \cup \stackrel{\circ}{h}$ and hence $q \leq h$.

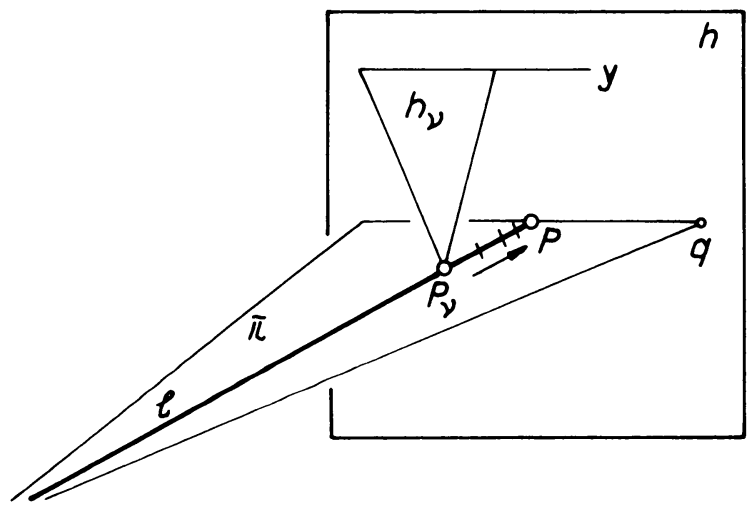

Figure 2 
Let $p \neq p_{\nu} \lessdot l$ be a generalized sequence converging to $p$. Then $h_{\nu}:=y \vee p_{\nu}$ is a generalized sequence of hyperplanes, which by $(\mathrm{CV})$ converges to $h=y \vee p$. For the plane $\pi:=l \vee q$ we have that $\pi \wedge h=p \vee q$ is a line. Hence by (S), $\pi \wedge h_{\nu}$ finally is a line, too. On the other hand, since $l$ is maximal regular also in the topological plane $\mathbf{G}_{\pi}, 3.4$ implies $\stackrel{\circ}{\pi}=\stackrel{\circ}{l} \cup\{q\}$. Since both $l<h_{\nu}$ and $q<h_{\nu}$ are impossible, $\pi \wedge h_{\nu}=p_{\nu}$ is a point. This is a contradiction.

Definition (cf. [CR 70, p. 12.1]; [Wh 86, p. 176]). A flat $x$ of a geometric lattice $\mathbf{G}$ is a separator if and only if for each "nonincident" pair $p \not \leq h$ of a point $p$ and a hyperplane $h$, either $p \leq x$ or $x \leq h$ holds.

In each $\mathbf{G}, 0$ is a separator. A separator $s$ is called minimal if and only if $s \neq 0$ and there exists no separator $s^{\prime}$ with $0<s^{\prime}<s$.

3.8. Theorem. Maximal regular flats $x$ are minimal separators.

Proof. Let $x$ be a maximal regular flat.

Claim: $x$ is a separator. If $x=1$, then trivially $x$ is a separator. If $x<1$, let $0 \lessdot p \not \leq h \lessdot 1$ be given. Assume $x \not \leq h$.

Case 1: $\operatorname{dim} x \geq 2$. Then $x \wedge h \neq 0$. There exists a hyperplane $h^{\prime}$ satisfying $0<x \wedge h^{\prime}<h^{\prime}$. By 3.6, we have ${ }_{0} \mathbf{G}=\stackrel{\circ}{x} \cup \stackrel{\circ}{h}^{\prime}$. Hence $x \wedge h=0$ would imply $\stackrel{\circ}{h} \subseteq \stackrel{\circ}{h^{\prime}}$, and therefore $h=h^{\prime}$, a contradiction to $x \wedge h^{\prime} \neq 0$. Now because of $0<x \wedge h<x, 3.6$ implies $p \leq x$.

Case 2: $x=: l$ is a line. Then by 3.7 we have $。 \mathbf{G}=\stackrel{\circ}{l} \cup \dot{y}$. From this one can easily determine the hyperplanes of $\mathbf{G}: l \not h$ implies $h=q \vee y$ for some $q \lessdot l$. Hence $p \not \leq h$ implies $p \leq l=x$.

Claim: The flat $x$ is minimal. For each $0<s<x$ we construct a pointhyperplane pair $0 \lessdot p \not \leq h \lessdot 1$ such that $p \not \leq s$ and $s \not \leq h$ : In the geometry $\mathbf{G}_{x}$, choose points $p \not \leq s$ and $q \leq s$. By $(*)$ the line $p \vee q$ contains a third point $r$. Again because of $(*)$, we may apply [Gr 86a, 2.3b] to $x=r, y_{1}=p$, $y_{2}=s$, and obtain a flat $k \lessdot x$ containing neither $p$ nor $s$. There exists a hyperplane $h \geq k$ of $\mathbf{G}$ not containing $x$.

\section{FACTORIZATION}

4.1. For each separator $s$, the set $\mathbf{G}_{s}$ of flats below $s$ is open.

Proof. By the definition of the topology on G (see $\S 1 . b .2$ ), we must show that for each $i$ the set ${ }_{i} \mathbf{G}_{s}$ of $i$-flats below $s$ is open in the set ${ }_{i} \mathbf{G}$ of all $i$-flats. We use induction on $i$ :

I. $i=0$ : By [CR 70, Proposition 12.4], G contains a complement $t$ of $s$ such that each point is either on $s$ or on $t$. Since the set $t$ of all points below any flat $t$ is closed [Gr 86a, 4.1], ${ }_{\circ} \mathbf{G}_{s}=\stackrel{\circ}{s}={ }_{0} \mathbf{G} \backslash{ }^{\circ} t$ is open.

II. Assume ${ }_{i} \mathbf{G}_{s}$ is open. Since ${ }_{i} \bigvee_{i+1}$ is an open function [Gr 86a, 4.9], ${ }_{i+1} \mathbf{G}_{s}={ }_{i} \mathbf{G}_{s} \stackrel{i+1}{\bigvee} \stackrel{\circ}{S}$ is also open.

4.2. Corollary. Each separator is isolated.

Proof. By 4.1, $\{s\}={ }_{\operatorname{dim} s} \mathbf{G}_{s}$ is open in $\operatorname{dim} s \mathbf{G}$.

Two flats $s, t$ are said to form a skew pair if they form a modular pair with $s \wedge t=0$; see e.g. [CR 70, p. 219]. 
4.3. If two flats $s$ and $t$ form a skew pair, then the restriction of $\vee$ to $\mathbf{G}_{s} \times \mathbf{G}_{t}$ is continuous.

Proof. Let $\left(x_{\nu}, y_{\nu}\right),(x, y) \in \mathbf{G}_{s} \times \mathbf{G}_{t}$, where $x_{\nu}$ and $y_{\nu}$ are generalized sequences converging to $x$ resp. $y$. Then $\operatorname{dim} x_{\nu}=\operatorname{dim} x=: i$ and $\operatorname{dim} y_{\nu}=$ $\operatorname{dim} y=: j$ finally. By [MM 70, 1.53], $s$ M implies $x_{\nu}$ M $y_{\nu}$. Together with $x_{\nu} \wedge y_{\nu}=0$ we obtain

$$
\operatorname{dim} x_{\nu} \vee y_{\nu}=\operatorname{dim} x_{\nu}+\operatorname{dim} y_{\nu}-\operatorname{dim} x_{\nu} \wedge y_{\nu}=i+j+1
$$

finally. Hence the continuity of ${ }_{i}^{j} \bigvee_{k}\left[\mathrm{Gr} 86 \mathrm{a}\right.$, Theorem 4.16] implies $x_{\nu} \vee y_{\nu} \rightarrow$ $x \vee y$.

We need the following extension of [CR 70; $§ 12$, Theorem 3] to topological geometries:

4.4. For two elements $s, t$ the map

$$
l_{s} \times l_{t}: \mathbf{G}_{s} \times \mathbf{G}_{t} \rightarrow \mathbf{G} \text {, defined by }(x, y) \mapsto x \vee y,
$$

is an isomorphism of topological geometries if and only if $s$ and $t$ are complementary separators.

Proof. Because of [CR 70; $§ 12$, Theorem 3] it suffices to show: Let $s$ and $t$ be complementary separators. If $l_{s} \times l_{t}$ is an isomorphism of geometric lattices, then it is also a homeomorphism.

Since $l_{s} \times l_{t}$ is bijective, it suffices to show that it is (1) continuous, and (2) open.

(1) Since $\mathbf{G}$ is isomorphic to $\mathbf{G}_{s} \times \mathbf{G}_{t}$ as a geometric lattice, we have

$$
\operatorname{dim} s \vee t=\operatorname{dim} s+\operatorname{dim} t+1,
$$

and hence (see e.g. [CR 70, Proposition 2.8]) $s \mathrm{M} t$. We now can apply 4.3.

(2) If $A$ is an open subset of $\mathbf{G}_{s}$, then by 4.1 it is also open in $\mathbf{G}$. Likewise for $B \subseteq \mathbf{G}_{t}$. Hence the openness of $\vee[\mathrm{Gr} 86 \mathrm{a}$, Corollary 4.14] implies that $A \vee B$ is open in $\mathbf{G}$.

4.5. Corollary. Denote by $r_{1}, \ldots, r_{k}$ the maximal regular flats of $\mathbf{G}$, and let $d$ be a complement of $r_{1} \vee \cdots \vee r_{k}=: s$. Then $d$ is unique, and the map

$$
\imath_{1} \times \cdots \times l_{k} \times \imath: \mathbf{G}_{r_{1}} \times \cdots \times \mathbf{G}_{r_{k}} \times \mathbf{G}_{d} \rightarrow \mathbf{G}
$$

defined by $\left(x_{1}, \ldots, x_{k}, y\right) \mapsto x_{1} \vee \cdots \vee y_{k} \vee y$ is an isomorphism of topological geometries.

Proof. By Theorem 3.8, each $r_{i}$ is a separator. By [CR 70, $\S 12$, Theorem 2] so is each join of these, in particular $s$. By [CR 70, Proposition 12.4] there is exactly one complement $d$ of $s$, and $d$ is again a separator. We now use 4.4 for $k=1,2,3, \ldots$ inductively.

4.6. Main Theorem I. Each topological geometry $\mathbf{G}$ is isomorphic to the direct product of finitely many

(1) open subgeometries of projective spaces over topological fields,

(2) topological planes (not necessarily desarguesian or projective),

(3) lines over Hausdorff topological spaces, or

(4) discrete geometries 
Proof. Because of 4.5 it suffices to know all regular topological geometries:

(1) For dimension at least three, these have been classified in [ $\mathrm{Gr} 86 \mathrm{~b}$, Corollary 4.5$]$.

(2), (3): Dimensions 2 resp. 1: see [Gr 86a, p. 115, Remarks 2 and 3] (here regularity is not needed).

\section{VARIETIES}

We now take over from Kahn and Kung, just replacing "finite" (combinatorial) by "topological", the following definition motivated in [KK 82, p. 486]:

Definition. A class $\mathscr{T}$ of topological geometries is called a variety if and only if

("hereditary") $\mathscr{T}$ is closed under the formation of isomorphic copies, (Sub) subgeometries, $(\mathrm{C})$ contractions, and (P) finite direct products; and

$\mathscr{T}$ contains a sequence $\mathbf{T}_{1}, \mathbf{T}_{2}, \mathbf{T}_{3}, \ldots$ ("universal models") (U) of dimensions $1,2,3, \ldots$ such that each $\mathbf{G} \in \mathscr{T}$ of dimension $i$ is isomorphic to a subgeometry of $\mathbf{T}_{i}$.

Remark. (Sub) means that a subgeometry is also in $\mathscr{T}$ provided it is a topological geometry again. Note that this is not always the case: Let $\mathbf{G}$ be the euclidean plane, and $M$ the closed unit disc. Then $\mathbf{G}(M)$ does not satisfy $(\mathrm{S})$.

Notation. Let $X, Y$ be two topological geometries (topological spaces, topological fields). We write

$$
X \prec Y
$$

if and only if $X$ is isomorphic to a subgeometry (subspace, subfield) of $Y$.

Remark. $X \prec Y$ and $Y \prec X$ do not imply $X \cong Y$ : For the topological spaces $X=\mathbb{R}$ and $Y=[0,1]$ we have $Y \prec X$ and $X \cong] 0,1[\prec Y$. Nevertheless $X \not \equiv Y$ (cf. $\S 1 . a$ ).

Definition. a. Projective variety $\mathscr{L}(F)$ : Let $F$ be a topological field. Define $\mathscr{L}(F)$ to be the class of all topological geometries isomorphic to some subgeometry of some topological projective $n$-space $\mathbf{P}_{n}(F)=: \mathbf{P}_{n}$ (see $\S 1$ ).

b. Matchstick variety $\mathscr{M}(X)$ : Let $X$ be a Hausdorff topological space containing at least two elements. Let $p \in X$. Define $\mathbf{l}:=$ topological line over $X$ (see [Gr 86a, p. 115, Remark 3]), and $\mathbf{p}:=\{0, p\}, 0 \neq p$, ("topological point"). Let $\mathbf{M}_{2 k-1}:=\mathbf{l}^{k}$, and $\mathbf{M}_{2 k}:=\mathbf{l}^{k} \times \mathbf{p}$. Define $\mathscr{M}(X)$ to be the class of all topological geometries isomorphic to some subgeometry of some $\mathbf{M}_{n}$.

We hasten to supplement:

5.1. Each $\mathscr{L}(F)$ and each $\mathscr{M}(X)$ is a variety.

Proof. Straightforward.

5.2 Lemma. Let $F, F^{\prime}$ be topological fields, and let $2 \leq m \leq n$ be integers. Then

$$
\mathbf{P}_{m}(F) \prec \mathbf{P}_{n}\left(F^{\prime}\right) \Leftrightarrow F \prec F^{\prime} .
$$


Proof. $(\Rightarrow)$ Because of $m \geq 2$ we can conclude $\mathbf{P}_{2}(F) \prec \mathbf{P}_{2}\left(F^{\prime}\right)=: \mathbf{P}$. Thus $\mathbf{P}_{2}(F) \cong \mathbf{P}(M)$ for some point set $M \subseteq{ }_{0} \mathbf{P}$. Since $\mathbf{P}$ induces on $M$ a (topological) projective plane, $M$ contains a quadrangle. Any corresponding ordered quadrangle $(o, u, v, i)$ induces operations + and $\cdot$ on the points $\neq v$ of the line $o \vee v=: l$, making it a (ternary) field $K^{\prime}$ (see e.g. [HP 73, V.2]; [Ha 59, 20.3]; [Pi 75, 1.5]). Since,$+ \cdot$, and ${ }^{-1}$ are multiple compositions of the functions $\circ \stackrel{1}{V}_{\circ}$ and ${ }^{1} \Lambda^{1}$, by $(\mathrm{C} \vee)$ and $(\mathrm{C} \wedge), K^{\prime}$ is a topological field. One verifies that each P-automorphism in $P G L_{2}\left(F^{\prime}\right)$ is continuous. Hence the transitivity of the group $P G L_{2}\left(F^{\prime}\right)$ on the set of ordered quadrangles (see e.g. [HP 73, Theorem 2.12]) implies $K^{\prime} \cong F^{\prime}$ (as topological fields). Furthermore, $\stackrel{\circ}{l} \cap M \backslash\{v\}$ becomes a subfield $K \leq K^{\prime}$. In $\mathbf{P}_{2}(F)$, the ordered quadrangle corresponding to $(o, u, v, i)$ under the isomorphism $\mathbf{P}(M) \cong \mathbf{P}_{2}(F)$ yields a topological field $K^{*} \cong K$. Analogously to the above, the transitivity of $P G L_{2}(F)$ implies $K^{*} \cong K$. To sum up, $F \cong K^{*} \cong K \leq K^{\prime} \cong F^{\prime}$ implies $F \prec F^{\prime}$.

$(\Leftarrow)$ Verification, using [ $\mathrm{Gr} 86 \mathrm{a}, 4.22]$.

5.3. Let $\mathscr{S}, \mathscr{T}$ be varieties containing sequences $\mathbf{S}_{n}$ resp. $\mathbf{T}_{n}$ of universal models such that there exists an integer $k$ with $\mathbf{S}_{n} \prec \mathbf{T}_{n}$ for all $n \geq k$. Then $\mathscr{S} \subseteq \mathscr{T}$.

Proof. (U), transitivity of $\prec$, (Sub). For $\operatorname{dim} \mathbf{G}<k$ : (P).

5.4. Main Theorem II. Let $\mathscr{T}$ be a variety of topological geometries containing a nondiscrete one. Then $\mathscr{T}$ is either

A. a projective variety $\mathscr{L}(F)$ over some topological field $F$, or

B. a matchstick variety $\mathscr{M}(X)$ over some Hausdorff topological space $X$.

Proof. Since $\mathscr{T}$ contains a nondiscrete geometry, by the Main Theorem I, [Gr $86 \mathrm{a}, 5.4(2) \Rightarrow(6)],(\mathrm{Sub})$, and $(\mathrm{U}), \mathrm{T}_{1}$ must be a regular line.

Case A: $\mathbf{T}_{2}$ regular. Then all $\mathbf{T}_{n}$ are regular (cf. [KK 82, Lemma 5]): From (Sub), (P) and (U) we deduce $\mathbf{T}_{1}, \mathbf{T}_{n-2}, \mathbf{T}_{n-1} \prec \mathbf{T}_{n}$. Assume $\mathbf{T}_{n}$ is not regular, but all preceding $\mathbf{T}_{i}$ are. Then the Main Theorem I and $\mathbf{T}_{n-1} \prec \mathbf{T}_{n}$, as well as the regularity of $\mathbf{T}_{n-1}$, imply $\mathbf{T}_{n} \cong \mathbf{H} \times \mathbf{p}$. Here $\mathbf{H}$ is a regular topological $(n-1)$-space, and $\mathbf{p}$ is a point. Now from $(\mathrm{P})$ and $(\mathrm{U})$ we get $\mathbf{T}_{n-2} \times \mathbf{T}_{1} \prec \mathbf{H} \times \mathbf{p}$. Because of the regularity of $\mathbf{T}_{n-2}$ and $\mathbf{T}_{1}$, this implies $\mathbf{T}_{n-2} \times \mathbf{T}_{1} \prec \mathbf{H}$, a contradiction to $\operatorname{dim} \mathbf{H}=n-1$.

The regularity of the $\mathbf{T}_{n}$ implies for $n \geq 3$, by [ $\mathrm{Gr} 86 \mathrm{~b}$, Corollary 4.5], the existence of a topological field $F_{n}$ with $\mathbf{T}_{n} \prec \mathbf{P}_{n}\left(F_{n}\right)$. Let $F=: F_{3}$. We will show $\mathscr{T}=\mathscr{L}(F)$ by a sequence of steps:

(1) $\mathbf{T}_{n}^{p} \cong \mathbf{P}_{n-1}\left(F_{n}\right)$ for each point $p$ of $\mathbf{T}_{n}$ : Since $\mathbf{T}_{n}$ may be embedded as an open subgeometry of $\mathbf{P}_{n}\left(F_{n}\right)=: \mathbf{P}$ [Gr 86b, Corollary 4.5], we have by [Gr 86a, Corollary 5.6c] that $\mathbf{T}_{n}^{p}=\mathbf{P}^{p} \cong \mathbf{P}_{n-1}\left(F_{n}\right)$.

(2) $F \prec F_{n}$ : Since $\prec$ is transitive, it suffices to show $F_{n} \prec F_{n+1}$ : Because of (C), (P), and (U) we have $\mathbf{T}_{n}^{p} \times \mathbf{T}_{1} \prec \mathbf{T}_{n+1} \prec \mathbf{P}_{n+1}\left(F_{n+1}\right)$. Together with (1) we deduce $\mathbf{P}_{n-1}\left(F_{n}\right) \prec \mathbf{P}_{n+1}\left(F_{n+1}\right)$. By Lemma $5.2(\Rightarrow)$ this implies $F_{n} \prec F_{n+1}$.

(3) $F_{n} \prec F$ : Because of (1), (C), and (U) we have $\mathbf{P}_{n-1}\left(F_{n}\right) \cong \mathbf{T}_{n}^{p} \prec \mathbf{T}_{n-1} \prec$ $\mathbf{P}_{n-1}\left(F_{n-1}\right)$. By Lemma $\left.5.2 \Leftrightarrow\right)$ this implies $F_{n} \prec F_{n-1}$.

(4) $\mathbf{P}_{n}(F) \prec \mathbf{P}_{n}\left(F_{n+1}\right) \cong \mathbf{T}_{n+1}^{p} \prec \mathbf{T}_{n}$ by (2), Lemma $5.2(\Leftarrow)$, (1), (C), and

(5) $\mathbf{T}_{n} \prec \mathbf{P}_{n}\left(F_{n}\right) \prec \mathbf{P}_{n}(F)$ by (3) and Lemma $5.2(\Leftarrow)$. 
(6) $\mathscr{T}=\mathscr{L}(F)$ because of (4), (5), and 5.3.

Case B: $\mathbf{T}_{2}$ not regular. Then by $(\mathrm{Sub})$ and $(\mathrm{U})$, no $\mathrm{T}_{n}$ contains a regular plane. For an arbitrary variety, $(\mathrm{P})$ and $(\mathrm{U})$ imply:

$$
\mathbf{M}_{2 k-1}:=\mathbf{T}_{1}^{k} \prec \mathbf{T}_{2 k-1} \text { and } \quad \mathbf{M}_{2 k}:=\mathbf{T}_{1}^{k} \times \mathbf{p} \prec \mathbf{T}_{2 k},
$$

where $\mathbf{p} \in \mathscr{T}$ is a point. Combining all this with the regularity of $\mathbf{T}_{1}$ and the Main Theorem I, we obtain

$$
\mathbf{T}_{2 k-1}=\mathbf{l}_{1,2 k-1} \times \cdots \times \mathbf{l}_{k, 2 k-1} \text { and } \mathbf{T}_{2 k}=\mathbf{l}_{1,2 k} \times \cdots \times \mathbf{l}_{k, 2 k} \times \mathbf{p},
$$

where $\mathbf{l}_{i j}$ are regular lines. (Sub) and (U) imply $\mathbf{l}_{i j} \prec \mathbf{T}_{1}$. Hence $\mathbf{T}_{n} \prec \mathbf{S}_{n}$. In summary, 5.3 implies $\mathscr{T}=\mathscr{M}(X)$, where $X$ is the point space of $\mathbf{T}_{1}$.

\section{ACKNOWLEDGMENTS}

The author would like to thank Professor J. P. S. Kung (North Texas State University) for communicating this problem to him, thus also promoting the general theory of topological geometries, and Professor $\mathrm{H}$. Mäurer (Technische Hochschule Darmstadt) for helpful suggestions.

\section{REFERENCES}

[Bi 67] G. Birkhoff, Lattice theory, 3rd ed., Amer. Math. Soc., Providence, R.I., 1967.

[CG 89] T. H. Choe and H. Groh, Topological geometric lattices, Abh. Math. Sem. Univ. Hamburg 59 (1989), 39-42.

[CR 70] H. H. Crapo and G.-C. Rota, On the foundations of combinatorial theory: Combinatorial geometries, M.I.T. Press, Cambridge, Mass., 1970.

[Du 66] J. Dugundji, Topology, Allyn \& Bacon, Boston, Mass., 1966.

[Gr 86a] H. Groh, Geometric lattices with topology, J. Combin. Theory Ser. A 42 (1986), 111-125.

[Gr 86b] _ Embedding geometric lattices with topology, J. Combin. Theory Ser. A 42 (1986), 126-136.

[Ha 59] M. Hall, Jr., The theory of groups, Macmillan, New York, 1959.

[HP 73] D. R. Hughes and F. Piper, Projective planes, Springer, New York, 1973.

[Ka 74] W. M. Kantor, Dimension and embedding theorems for geometric lattices, J. Combin. Theory Ser. A 17 (1974), 173-195.

[Ku 86] J. P. S. Kung, A source book in matroid theory, Birkhäuser, Boston, Mass., 1986.

[KK 82] J. Kahn and J. P. S. Kung, Varieties of combinatorial geometries, Trans. Amer. Math. Soc. 271 (1982), 485-499.

[Mi 68] J. Misfeld, Topologische projektive Räume, Abh. Math. Sem. Univ. Hamburg 32 (1968), 232-263.

[MM 70] F. Maeda and S. Maeda, Theory of symmetric lattices, Springer, Berlin, 1970.

[Pi 75] G. Pickert, Projektive Ebenen, 2nd ed., Springer, Berlin, 1975.

[Sz 86] H. Szambien, Topological projective geometries, J. Geometry 26 (1986), 163-171.

[We 76] D. J. A. Welsh, Matroid theory, Academic Press, London, 1976.

[Wh 86] N. White (Editor), Theory of matroids, Cambridge Univ. Press, Cambridge (GB), 1986.

[Wh 87] _ Combinatorial geometries, Cambridge Univ. Press, Cambridge (GB), 1987.

Fachbereich Mathematik, Technische Hochschule Darmstadt, D-W-6100 Darmstadt, GERMANY 